\title{
The Large Ultraviolet/Optical/Infrared Surveyor
}

\author{
LUVOIR is a concept for a powerful, flexible space observatory to enable the first survey for exoplanets most \\ similar to the Earth, search for signs of life in our Solar System and beyond, and revolutionize astrophysics in the \\ twenty-first century.
}

\section{Aki Roberge and Leonidas A. Moustakas}

A stronomy crossed a threshold three decades ago with the discovery of planets around other stars $^{1,2}$. Compared to scientists' previous expectations set by the Solar System, exoplanets are wonderfully abundant and varied. Indirect planet discovery techniques have shown that small rocky planets residing in stellar habitable zones, where such planets may have liquid water on their surfaces, are not rare. This revelation drives us to ask more ambitious and fundamental questions that fascinate scientists and the public alike: are there other truly Earth-like planets out there and do any of them harbour life?

Today, exoplanets are largely 'small black shadows' to us, with measurements of orbits, sizes and masses (all three in the best cases). The upcoming James Webb Space Telescope and future 30-m-class ground-based telescopes will characterize the atmospheres of habitable planet candidates orbiting low-mass $M$ dwarf stars ${ }^{3,4}$. However, deeply probing atmospheres of the exoplanets most similar to the Earth, those around Sun-like stars, remains out of reach for currently planned observatories. Bringing them within our grasp is a primary motivation for the Large UV/Optical/Infrared Surveyor (LUVOIR) mission concept, currently the focus of a three-year NASA study.

LUVOIR is a space telescope in the tradition of NASA's Great Observatories, with diverse and powerful capabilities to revolutionize the astrophysics landscape. The view of the Science and Technology Definition Team (STDT) directing the study is that a broad, community-driven observing programme would make best scientific use of LUVOIR's capabilities. LUVOIR is being designed to be serviceable and upgradable, features that greatly contributed to the unparalleled success of the Hubble Space Telescope (HST). LUVOIR aims to return the highest benefit-to-cost ratio from a large mission with a long development timescale, with the flexibility to respond to future discoveries and answer questions the scientific community hasn't yet conceived.

The two LUVOIR designs being studied have five-year primary missions and a possible

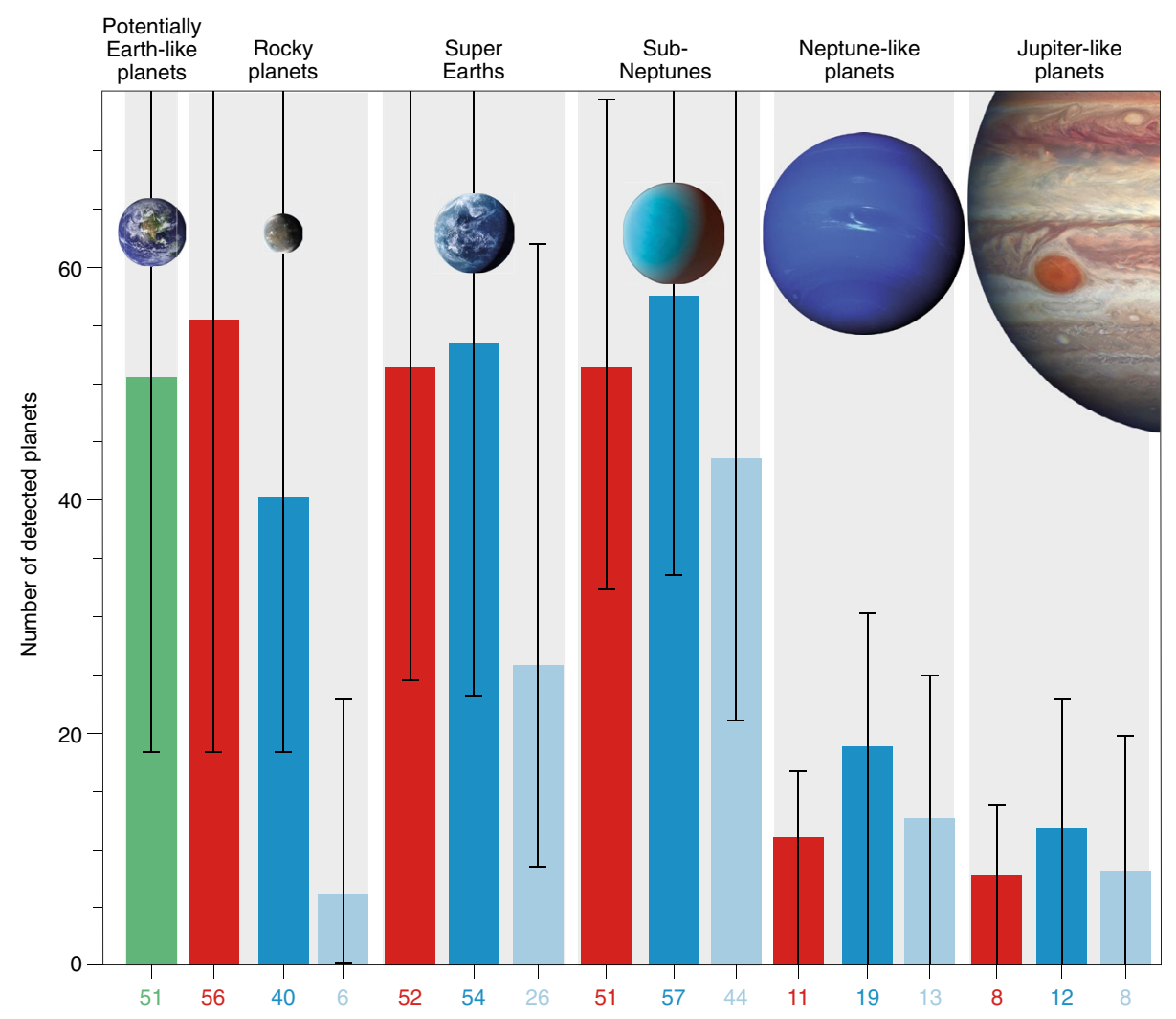

Fig. 1 | Expected exoplanet detection yields with the LUVOIR-A concept. Planet classes from left to right are: habitable planet candidates (green bar), rocky planets, super-Earths, sub-Neptunes, Neptunes and Jupiters. Red, blue and ice blue bars indicate hot, warm and cold planets, respectively. The habitable planet candidates are a subset of the warm rocky and super-Earth planets. All these different exoplanets are expected to be detected during the habitable planet search campaign. Credit: C. Stark (STSCl); Earth-like, NASA; rocky, NASA/Ames/JPL-Caltech; super-Earth, ESO/M. Kornmesser/Nick Risinger (http://skysurvey.org/); sub-Neptunes, NASA/JPL-Caltech/R. Hurt (SSC); Neptune-like, NASA/JPL; and Jupiter-like, NASA/ESA/A. Simon (NASA Goddard).

launch date in the late 2030s. The first design (LUVOIR-A) has a 15-m diameter primary telescope mirror designed for launch on NASA's future Space Launch System rocket. The second (LUVOIR-B) has a roughly 8-m diameter primary mirror intended for launch in a heavy-lift rocket similar to those in use today. The two architectures explore the scalability of the LUVOIR segmented telescope design in science return, design complexity, technical risk and cost.
Four instruments are being designed for LUVOIR-A. The Extreme Coronagraph for Living Planetary Systems (ECLIPS) is designed for ultrahigh-contrast $\left(10^{-10}\right)$ observations: imaging (from $200 \mathrm{~nm}$ to $1,030 \mathrm{~nm}$ ), integral field spectroscopy ( $R=140$ from $515 \mathrm{~nm}$ to $1,030 \mathrm{~nm}, R=70$ from $1,000 \mathrm{~nm}$ to $2,000 \mathrm{~nm}$ ), and pointsource spectroscopy $(R=200$ from 1,000 $\mathrm{nm}$ to $2,000 \mathrm{~nm}$ ). The LUVOIR Ultraviolet Multi-Object Spectrograph (LUMOS) will 


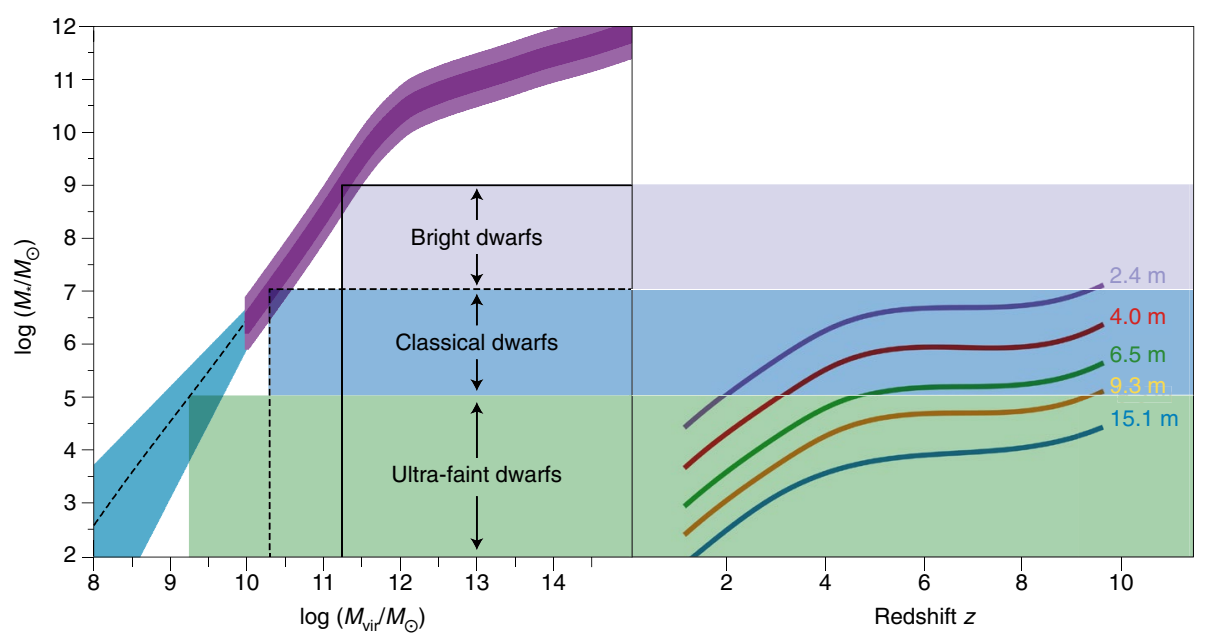

Fig. 2 | LUVOIR's view of the least luminous galaxies, the building blocks of galaxy formation, extends to eras when the Universe was just $3 \%$ of its current age. This corresponds to the $z=10$ point in the right panel, which shows the galaxy mass detection limits for LUVOIR-type architectures, including the 15.1-m LUVOIR-A. $M_{*}$ is the galaxy mass in stars and $M_{\text {vir }}$ is the galaxy halo (virial) mass. Credit: left panel adapted from ref. ${ }^{8}$, Annual Reviews; right panel M. Postman (STScl).

provide imaging from $100 \mathrm{~nm}$ to $200 \mathrm{~nm}$ and multi-object, multi-resolution $(R=500-60,000)$ spectroscopy from $100 \mathrm{~nm}$ to $400 \mathrm{~nm}$. The High Definition Imager $(\mathrm{HDI})$ is a wide field $\left(2^{\prime} \times 3^{\prime}\right)$ camera covering $200 \mathrm{~nm}$ to $2,500 \mathrm{~nm}$, Nyquist sampled at $400 \mathrm{~nm}$. Finally, POLLUX is a high-resolution $(R=120,000)$ point-source spectropolarimeter covering $97 \mathrm{~nm}$ to $390 \mathrm{~nm}$; this instrument is being designed by a consortium of European institutions, with support from the French space agency (CNES). Variants of the first three instruments are being studied for LUVOIR-B.

LUVOIR's science cases stretch from the dawn of the Universe to observations of Solar System worlds. Below we highlight just three of LUVOIR's 'signature science cases'. The LUVOIR Interim Report showcases a dozen signature science cases that represent compelling observing programmes scientists might carry out with LUVOIR at the limits of its performance. As exciting as these are, they are surely an incomplete vision of LUVOIR's future scientific potential.

\section{Exoplanets and the search for life}

To determine which exoplanets are actually habitable, we must measure the molecular constituents of rocky planet atmospheres, including water vapour and other greenhouse gases. Making these measurements for Earth-size planets around Sun-like stars demands analysing light from the planets themselves via direct spectra ${ }^{5,6}$, which is challenging due to the faintness of the planets and their close proximity to their host stars. Direct observations of rocky exoplanets around Sun-like stars demand the ultrahigh contrast possible only with a space-based telescope coupled to a highperformance starlight suppression system.

Measuring the frequency of Earthlike conditions on rocky worlds requires surveying hundreds of stars to find dozens of candidate exoplanets for study. This goal is a strong driver of telescope aperture size but guarantees that whatever is found will have a major scientific impact. In the absence of water detections, we would know that global-scale surface oceans are rare on rocky worlds in the habitable zone.

On the other hand, if we find that Earth-like conditions are common on rocky worlds near our Sun, then a stunning vista of hospitable new worlds will be unveiled.

While LUVOIR delivers the habitableplanet census, it will study hundreds of other types of planets, vastly expanding the scope of comparative planetology

(Fig. 1). It will also probe the atmospheres of habitable planets for biosignature gases and might find the first signs of life outside the Solar System. The spectrum of the Earth contains features arising from gases of biological origin, including oxygen and methane. By targeting exoplanets with sizes and orbits similar to Earth's and host stars similar to the Sun, we hope to increase our chances of finding - and recognizing extraterrestrial life.

\section{The births and deaths of galaxies} Throughout their lives, material flows into and out of galaxies as they interact with neighbouring galaxies and the intergalactic medium ${ }^{7}$. Some galaxies are born small but become the building blocks of giant spirals like our own Milky Way ${ }^{8}$. Some galaxies are actively forming new stars, while others have undergone slow stagnation as their stars age and no new ones are born. Our current understanding of this complex interplay is highly incomplete.

Much of the cycling of matter to, from and within galaxies is currently unobserved, as the gas flows are hot and tenuous. Ultraviolet spectroscopy, which must be done from space given the opacity of Earth's atmosphere below $300 \mathrm{~nm}$, can reveal these processes. Furthermore, galaxy mergers result in bursts of star formation but may also be responsible for the ultimate 'death' of merged galaxies. LUVOIR can illuminate the role mergers play in forming today's massive galaxies by enabling deep, high-resolution imaging of all types of galaxies across cosmic time. The smallest building blocks of galaxies become visible to LUVOIR at a time when the Universe was only $3 \%$ of its current age (Fig. 2).

\section{Monitoring the Solar System}

The search for life also takes place closer to home. Some moons of the outer Solar System have liquid water beneath their icy surfaces. These sub-surface oceans must be heated from below, which may also provide the energy needed for life. Ultraviolet imaging of Europa with Hubble has revealed aurorae produced by geysers emanating from the surface, possibly allowing glimpses into the deep ocean' (Fig. 3). LUVOIR can provide unique high-resolution UV spectral imaging of icy moons with high temporal cadence over several years. This will reveal the currently unknown strength and frequency of geyser activity, valuable supporting information for future spacecraft
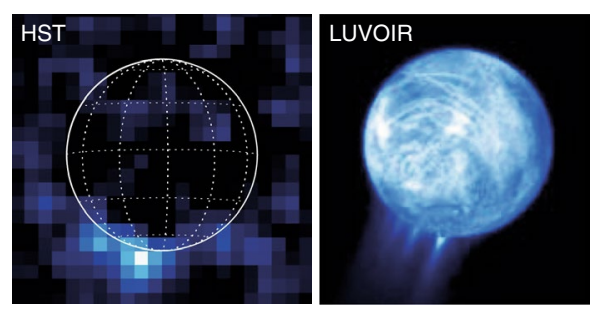

Fig. 3 | Spectroscopic imaging of Europa and its geysers. The left panel shows an aurora on Europa observed with $\mathrm{HST}^{7}$. This UV hydrogen emission (Lyman- $\alpha$ ) comes from dissociation of water vapour in geysers emanating from the surface. The right panel shows a simulation of how hydrogen emission from Europa would look to LUVOIR-A. The moon's surface is bright due to reflected solar Ly- $\alpha$ emission. Credit: G. Ballester (LPL). 
visiting these other ocean worlds to search for signs of life.

\section{The future of astrophysics}

The USA astrophysics community determines its future scientific priorities for both ground- and space-based research via Decadal Surveys managed by the USA's National Research Council. Unfortunately, the top priorities in the large space mission class from the last two Astrophysics Decadal Surveys are still on the ground: the James Webb Space Telescope and the Wide Field Infrared Survey Telescope (WFIRST). The reasons for this state of affairs are far beyond the scope of this Comment, but the consequences for astrophysics, as well as LUVOIR and other future large space observatory concepts, must be addressed.

It is important to acknowledge the vital role large missions play in a balanced scientific portfolio. Powerful, generalpurpose observatories are by their very nature especially well-suited to enabling unexpected scientific advances, which are often the most revolutionary. Furthermore, they serve the largest number of scientists across the widest range of fields. For many astronomers in the USA, NASA's Great Observatories have been their most important community facilities for decades $^{10}$.
The argument has been made that long development timescales and cost overruns on large missions harm NASA's ability to execute smaller, more focused missions, which would also be damaging to a balanced scientific portfolio. Examination of historical NASA Astrophysics budget data suggests that 'flagships eat flagships', not smaller missions (https://go.nature.com/2tUYAN7). However, restraining development times and costs on future large missions is still a critical goal.

Some helpful steps are being investigated within the LUVOIR study. Easily repairable, upgradable, and scalable observatory concepts can better respond to uncertain technological and budgetary landscapes. Technology gaps should be filled much earlier in the mission development process. Important products of the LUVOIR study will be a thorough assessment of technological maturity and a technology development plan that includes estimated schedule and cost, all of which will be independently checked. Finally, an observatory like LUVOIR can and should draw new resources to space astrophysics, through expanded international partnerships and nontraditional partnerships with other scientific and technical disciplines. But the greatest potential source of support comes from the public and their representatives in government. Ambitious and revolutionary science goals - like those of LUVOIR - are the way to win and sustain that support.

Aki Roberge ${ }^{1 *}$ and Leonidas A. Moustakas ${ }^{2}$ ${ }^{1}$ NASA Goddard Space Flight Center, Greenbelt, MD, USA. ${ }^{2} J e t$ Propulsion Laboratory, California Institute of Technology, Pasadena, CA, USA.

*e-mail: aki.roberge@nasa.gov

Published online: 1 August 2018

https://doi.org/10.1038/s41550-018-0543-8

References

1. Campbell, B., Walker, G. A. H. \& Yang, S. Astrophys. J. 331, 902-921 (1988).

2. Mayor, M. \& Queloz, D. Nature 378, 355-359 (1995).

3. Meadows, V. S. et al. Astrobiology 18, 133-189 (2018).

4. Wang, J., Mawet, D., Ruane, G., Hu, R. \& Benneke, B. Astron. J. 153, 183 (2017)

5. Kaltenegger, L. \& Traub, W. A. Astrophys. J. 698, 519-527 (2009).

6. Snellen, I. et al. Astron. Astrophys. 576, A59 (2015).

7. Tumlinson, J., Peeples, M. S. \& Werk, J. K. Annu. Rev. Astron. Astrophys. 55, 389-432 (2017).

8. Bullock, J. S. \& Boylan-Kolchin, M. Annu. Rev. Astron. Astrophys. 55, 343-387 (2017).

9. Roth, L. et al. Science 343, 171-174 (2014).

10. National Academies of Sciences, Engineering, and Medicine Powering Science: NASA's Large Strategic Science Missions (National Academies Press, Washington DC, 2017); https://doi. org/10.17226/24857

\section{Acknowledgements}

L.A.M.'s work was carried out at the Jet Propulsion Laboratory, California Institute of Technology, under a contract with the National Aeronautics and Space Administration. This article contains pre-decisional information and is for planning and discussion only. 